\title{
RELATIVISTIC HEAVY-ION PHYSICS WITHOUT NUCLEAR CONTACT
}

\section{The large electromagnetic field generated by a fast heavy nucleus allows investigation of new electromagnetic processes not accessible with real photons.}

\section{Carlos Bertulani and Gerhard Baur}

An increasing number of physicists are investigating nuclear collisions at relativistic energies. (See figure 1.) Accelerators completely devoted to the study of these collisions (such as the Relativistic Heavy Ion Collider at Brookhaven National Laboratory) are under construction. So are hadron colliders (such as the Large Hadron Collider at $\mathrm{CERN}$ ), which can also be used to accelerate heavy ions. ${ }^{1}$ The aim of these projects is to study what happens to nuclear matter at high pressures and temperatures. The expectation is that such experiments will access information that can test important predictions of quantum chromodynamics-for example, a nuclear matter transition from a mixture of quarks and gluons to hadrons, as occurred in the first moments of the universe according to the Big Bang theory. ${ }^{2}$

Relativistic heavy-ion collisions can also be an important tool for traditional nuclear physics research-for example, for investigating nuclear structure, hadronic structure, atomic physics and stellar nucleosynthesis. ${ }^{3}$ This has become more evident in the last few years as an increasing number of experiments have been carried out for such purposes. These experiments are based on what happens in distant collisions between relativistic nuclei, without nuclear contact. They are therefore very different on physical grounds from the frontal collisions referred to above. Whereas for central collisions the driving forces are mainly of hadronic origin, distant collisions without nuclear contact are completely dominated by electromagnetic interactions.

The electromagnetic interactions are so important in high-energy collisions between heavy ions primarily because of the ions' large charge (which means that the cross sections are enhanced by the large factors of $Z^{2}$ and $Z^{4}$ for one- and two-photon processes, respectively) and the Lorentz contraction. (See figure 2.) Lorentz contraction implies that in a collision with impact parameter $b$ the field generated by the relativistic nucleus with velocity $v$ is effective during a time interval $\Delta t$ given approxi-

Carlos Bertulani is a visiting scientist at the Gesellschaft für Schwerionenforschung, in Darmstadt, Germany, and a professor of physics at the Federal University of Rio de Janeiro, in Brazil. Gerhard Baur is a member of the scientific staff at the Forschungszentrum Jülich, in Jülich, Germany, and a professor of physics at the University of Basel, in Basel, Switzerland. mately by $b / \gamma v$ and that the electric (or magnetic) field during this time interval is very intense: $E \simeq \gamma Z e / b^{2}$. The factor $\gamma$, which is $\left(1-v^{2} / c^{2}\right)^{-1 / 2}$, is very large (on the order of $10^{4}-10^{7}$ ) in relativistic heavy-ion colliders.

\section{Theory}

In 1924 Enrico Fermi had an idea that led to what is now known as the Weizsäcker-Williams method (due to the improvements obtained by Carl Friedrich von Weizsäcker and Evan James Williams). ${ }^{4}$ Fermi calculated the effect of the time-dependent electromagnetic field of a fast charge, replacing it with an equivalent pulse of real photons. One can calculate the pulse of equivalent photons classically, and this method of using equivalent photons is described in textbooks of classical electrodynamics. ${ }^{5}$ With the advent of heavy-ion accelerators with relativistic energies-for example, the Bevalac at Lawrence Berkeley Laboratory-it became clear that heavy-ion physics without nuclear contact could be very useful in nuclear physics. Because the electromagnetic interaction is well known, the results of the experiments could be compared with the theoretical calculations and used to test models for photonuclear interaction and photon-photon interaction.

For a collision without nuclear contact, the electric field generated by one of the ions is divergence free in the interior of the other nucleus. One consequence of this is that the matrix elements involved in the nuclear excitation process are the same as those involved in photonuclear reactions. The cross sections for all such electromagnetic processes in heavy-ion collisions can then be written in a factorized form as

$$
\sigma=\sum_{\lambda} \int \frac{\mathrm{d} \omega}{\omega} n_{\lambda}(\omega) \sigma_{\gamma}^{\lambda}(\omega)
$$

Here $\hbar \omega$ is the excitation energy, $\sigma_{\gamma}^{\lambda}$ is the photonuclear cross section for a given multipolarity, and the $n_{\lambda}$ are virtual photon numbers, or equivalent photon numbers. The total photonuclear cross section is a sum of the contributions from all the multipolarities: $\sigma_{\gamma}=\Sigma \sigma_{\gamma}^{\lambda}$. Equation 1 contains the spirit of Fermi's method, but it enables one to calculate the equivalent photon numbers more accurately and for all multipolarities. ${ }^{3,6}$

The interpretation of equation 1 is that the timedependent field of a relativistic heavy ion has the same effect on a target nucleus as would a pulse of real 


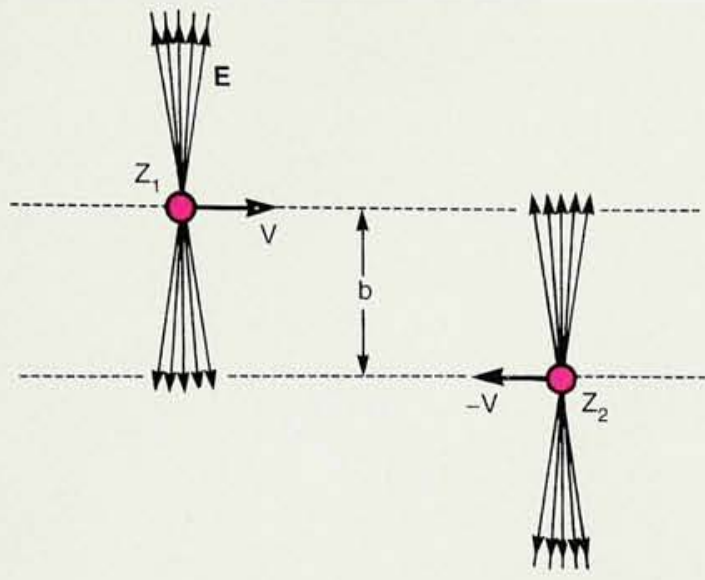

b

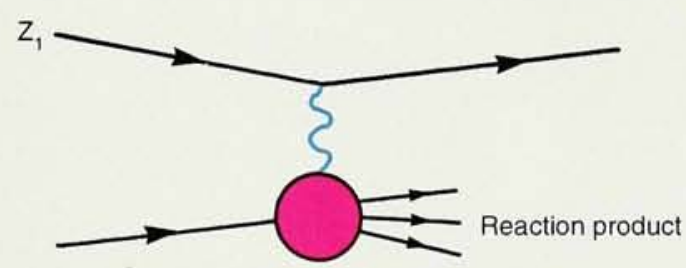

c

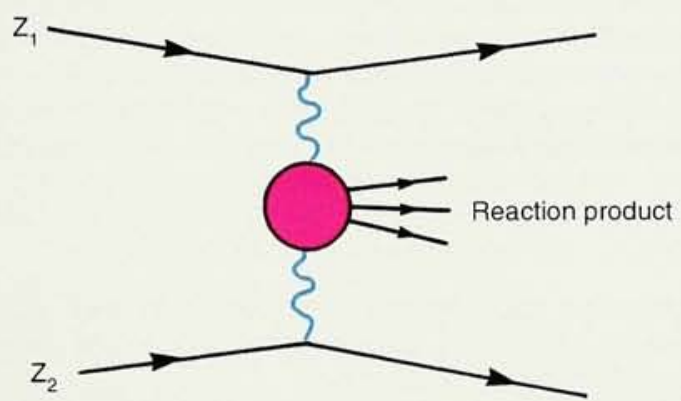

Enhanced interaction. The Lorentz contraction (a) of the intense fields generated by relativistic heavy ions can lead to very large cross sections for photonuclear processes (b) and for two-photon collision processes (c). Figure 2

$\gamma+{ }^{6} \mathrm{Li} \rightarrow \alpha+\mathrm{d}$, at a fixed energy $\omega$, are proportional. The cross section for the latter reaction, in turn, is proportional to that of the radiative capture reaction in accord with the detailed balance theorem. Phase-space considerations favor the Coulomb (or electromagnetic) dissociation method over direct measurements. ${ }^{8}$

The most suitable beam energies for performing such experiments seem to be around $100 \mathrm{MeV} /$ nucleon. These energies are available at the Grand Accelerateur National d'Ions Lourds (GANIL), in Caen, France; at the Gesellschaft für Schwerionenforschung (GSI, the Institute for Heavy-Ion Research), in Darmstadt, Germany; at Michigan State University, in East Lansing; and at the Institute of Physical and Chemical Research (RIKEN), in Wakoshi, Saitama, Japan. The first measurements using this technique are encouraging. ${ }^{9,10}$ Figure 3 shows data from two of these experiments. The cross sections in figure $3 \mathrm{a}$ are for the radiative capture reaction ${ }^{13} \mathrm{~N}(\mathrm{p}, \gamma){ }^{14} \mathrm{O}$ and were obtained with the Coulomb dissociation method using lead targets. ${ }^{9}$ The experiment obtained the value
$\Gamma_{\gamma}=3.1 \pm 0.6 \mathrm{MeV}$ for the radiative width of the $1^{-}$state in ${ }^{14} \mathrm{O}$ at the excitation energy $5.17 \mathrm{MeV}$. This radiative capture reaction is of great interest because it determines conditions for starting the hot CNO cycle rather than the regular $\mathrm{CNO}$ cycle. ${ }^{7}$ Figure $3 \mathrm{~b}$ shows data from another Coulomb dissociation experiment ${ }^{10}$ (colored points), for the radiative capture reaction ${ }^{4} \mathrm{He}(\mathrm{d}, \gamma)^{6} \mathrm{Li}$. These data were the first to be obtained in the low-energy region, below the resonance at $0.711 \mathrm{MeV}$. The other data (black points) were obtained in direct capture measurements. The solid curve is a theoretical calculation.

\section{Radioactive beams}

Studies of short-lived nuclei are now possible with heavyion accelerators and the development of new detection techniques. (See the article by Richard N. Boyd and Isao Tanihata in PHYSICS TODAY, June 1992, page 44.) The radioactive nuclei are produced in fragmentation reactions and further gathered in a secondary beam. Some short-lived nuclei are so weakly bound that they fragment when they collide with a target nucleus at a very large impact parameter. This results in huge cross sections for the electromagnetic dissociation of these nuclei. ${ }^{3}$ Many experiments of this type have been performed at GANIL, GSI, MSU, RIKEN and other accelerators.

An MSU experiment on the electromagnetic dissociation of ${ }^{11} \mathrm{Li}$ is a good example. ${ }^{11}$ The two-neutron separation energy of this nucleus is $340 \pm 50 \mathrm{keV}$, which is very small compared with the normal $6-8-\mathrm{MeV}$ binding energy of nucleons in nuclei. As a consequence, the last two neutrons in ${ }^{11} \mathrm{Li}$ are distributed in a large extension of radius about 8 femtometers outside a ${ }^{9} \mathrm{Li}$ core. The structure and reaction mechanisms for this and other radioactive nuclei are not very well known. It is not clear, for example, if the excitation and decay of this nucleus proceeds via an intermediate state or if it is a direct breakup transition. The MSU experiment seems to indicate that the latter possibility prevails. Figure $4 \mathrm{a}$ plots a sample of the data obtained in that experiment in the form of the decay spectrum $\mathrm{d} \sigma / \mathrm{d} E_{\mathrm{d}}$, which is proportional to the integrand of equation 1 and is directly related to the dipole response function $|\langle f|| x|| i\rangle|^{2}$ of the nucleus for a given excitation energy. If the width of this function is to be associated with the lifetime of a resonant state, the nucleus must decay far away from the target. However, a large shift in the energy of the ${ }^{9} \mathrm{Li}$ fragments (the Coulomb reacceleration effect) is observed, which is inconsistent with the existence of a resonant state. This is shown in figure $4 \mathrm{~b}$ for the difference in the velocities of the ${ }^{9} \mathrm{Li}$ and neutron fragments. According to the investigators in that experiment the peak in figure $4 \mathrm{a}$ should not be interpreted as the signature of a resonance. ${ }^{11}$ In fact, it can be explained from phase-space considerations alone. ${ }^{3}$

\section{Giant resonances}

From table 1 we see that the excitation of giant resonances in heavy-ion collisions does not require very-highenergy accelerators. In fact heavy-ion excitation of giant resonances is now an important field of research at GANIL, GSI, MSU and RIKEN. A very new topic for nuclear structure investigation is the electromagnetic excitation of multiphonon resonances-for example, a giant resonance excited on top of another one. ${ }^{12,13}$ This kind of excitation cannot be achieved in electron scattering or real photon experiments. But highly energetic heavy ions can be viewed as intense sources of virtual photons, and the probability of multiphoton exchange is not small.

Experiments aiming at multiphonon excitation have recently been performed at GSI. ${ }^{14,15}$ The experimenters 

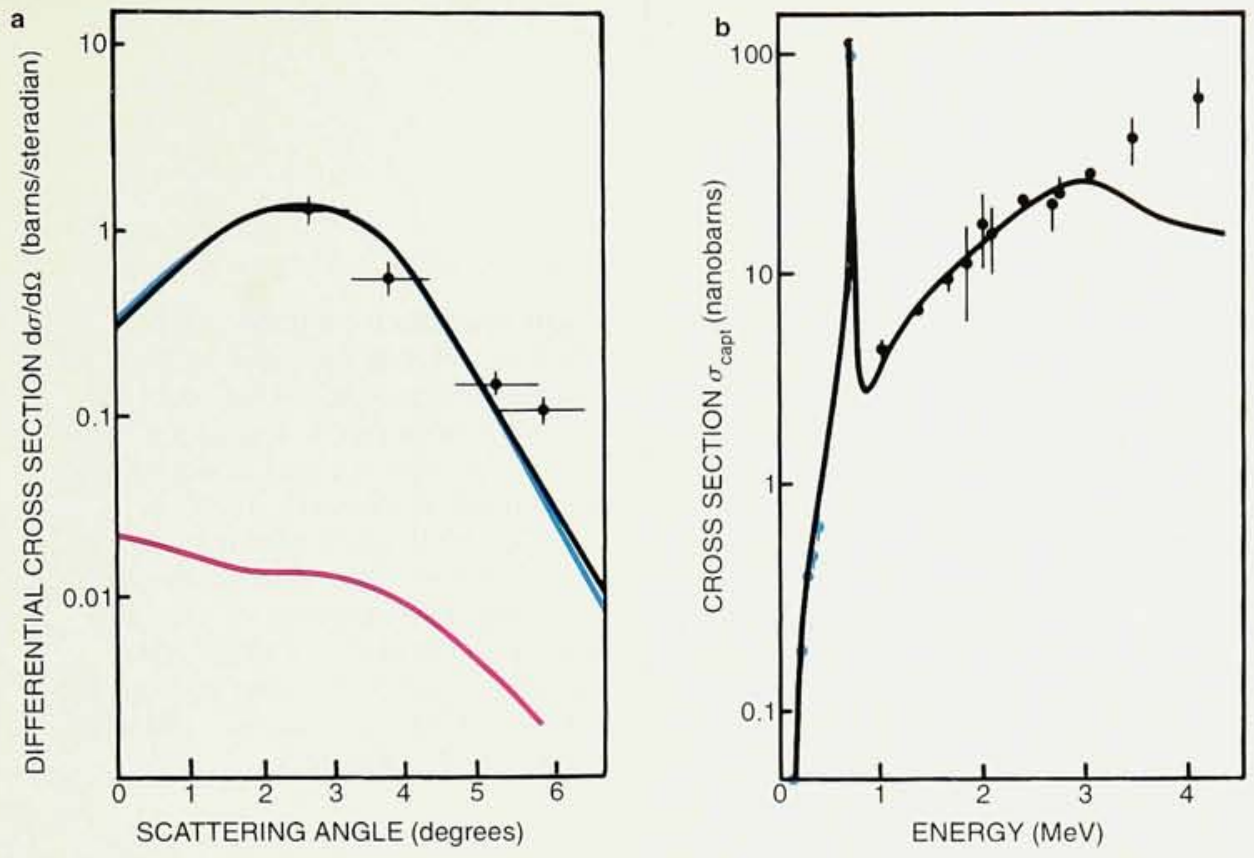

Electromagnetic excitation cross sections. a: Differential cross sections of the $1^{-}$resonant state in ${ }^{14} \mathrm{O}$ as a function of the center-of-mass scattering angle of the ${ }^{14} \mathrm{O}-{ }^{208} \mathrm{~Pb}$ system. The upper and lower colored curves represent the respective contributions of the Coulomb and nuclear interactions to the excitation. This experiment yielded, with good accuracy, the radiative width of the state, a quantity of great importance in nuclear astrophysics. (Adapted from ref. 9.) b: Radiative capture cross section for the reaction $\mathrm{d}(\alpha, \gamma)^{6} \mathrm{Li}$ obtained after a theoretical analysis of results similar to those shown in a. An experiment using the Coulomb dissociation method gave the data represented by the colored points. (Adapted from ref. 10.) Figure 3

identified the giant resonance states by measuring their gamma or particle decays. Figure 5 shows a sample of the data. Usual giant resonances are easily identified in these data at their expected positions and widths. A bump appearing at about twice the energy of the isovector giant dipole resonance was identified as a double-phonon state. More studies of the strengths, widths and other properties of multiphonon states using the electromagnetic excitation technique are expected to give important information on novel collective vibrations in nuclei.

With increasing energy of the heavy ions we can obtain even higher excitation energies. At the energies available at the Super Proton Synchrotron at CERN, one would expect that delta resonances could be excited electromagnetically. The deltas are nucleonic excitations of about $300 \mathrm{MeV}$. The decay of such a state to a nucleon and a pion releases about $300 \mathrm{MeV}$ of energy inside the nucleus. The reabsorption of the pion in the nucleus initiates a complicated cascading process that is of much interest for nuclear physics studies. If one uses veto detectors, which make it possible to separate out the nuclear contribution to the process, the electromagnetic excitation of multiple deltas in the nuclei also could be measured. Other useful cases, such as the electromagnetic production of hypernuclei, can also be studied in relativistic heavy-ion colliders.

The excitation of a single hadron in the strong electromagnetic field of a heavy nucleus is also a powerful method for deducing important features of these particles. A well-known example is the excitation of a $\Lambda$ into a $\Sigma^{0}$. The electromagnetic cross section for this process has been measured at CERN ${ }^{16}$ and at Fermilab ${ }^{17}$ using nickel and uranium targets and relativistic $\Lambda$ beams. The $\Sigma^{0}$ is a magnetic dipole excitation of the $\Lambda$, with an $80-\mathrm{MeV}$

Electromagnetic breakup. a: Cross section for the electromagnetic breakup of ${ }^{11} \mathrm{Li}$ projectiles as a function of the final relative kinetic energies of the fragments. The curve is a guide to the eye. (Adapted from ref. 11.) b: Distribution of differences in velocity between the ${ }^{9} \mathrm{Li}$ fragments and the two neutrons after the breakup. The histogram shows the result that would be expected if Coulomb reacceleration were not present. Figure 4 excitation energy. Before the introduction of the quark model, the magnetic dipole moment for this transition was calculated using the electromagnetic symmetry properties of the hadronic octuplets. The lifetime of the $\Sigma^{0}$

a

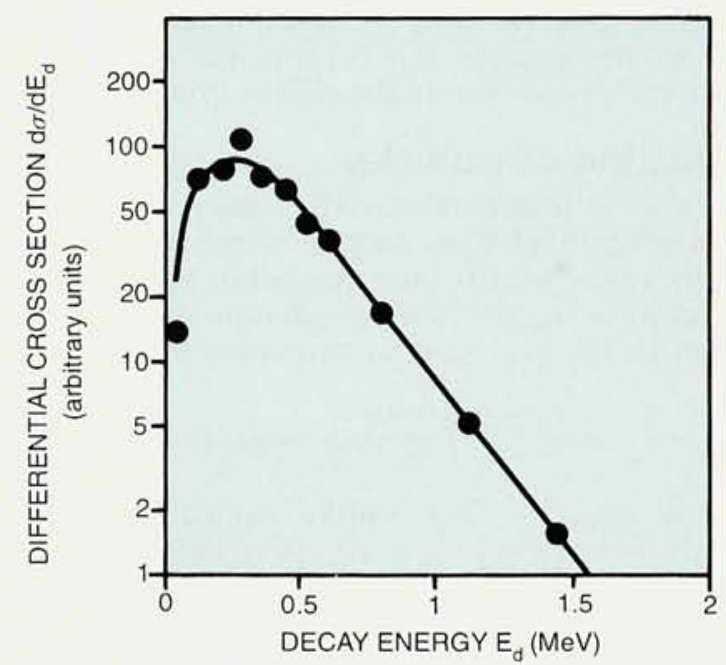

b

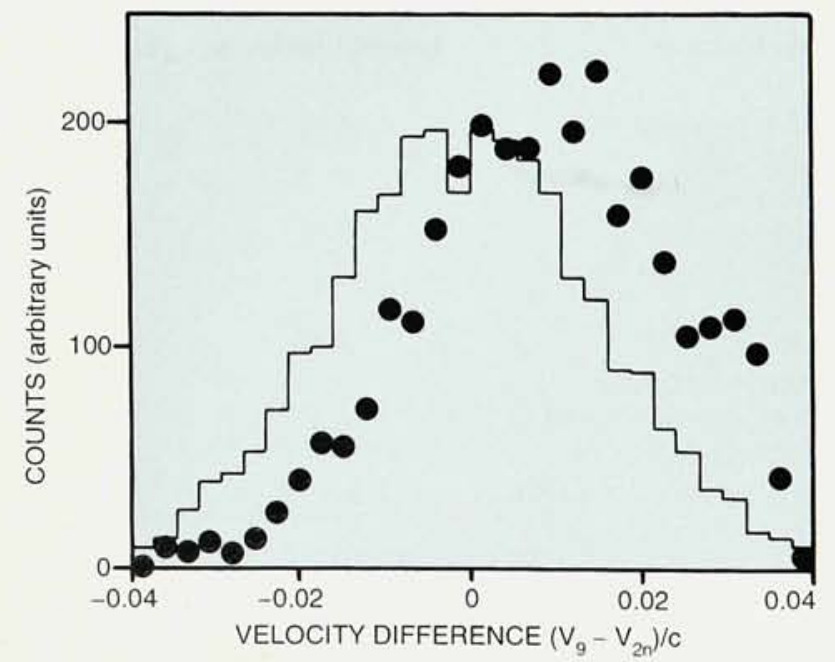




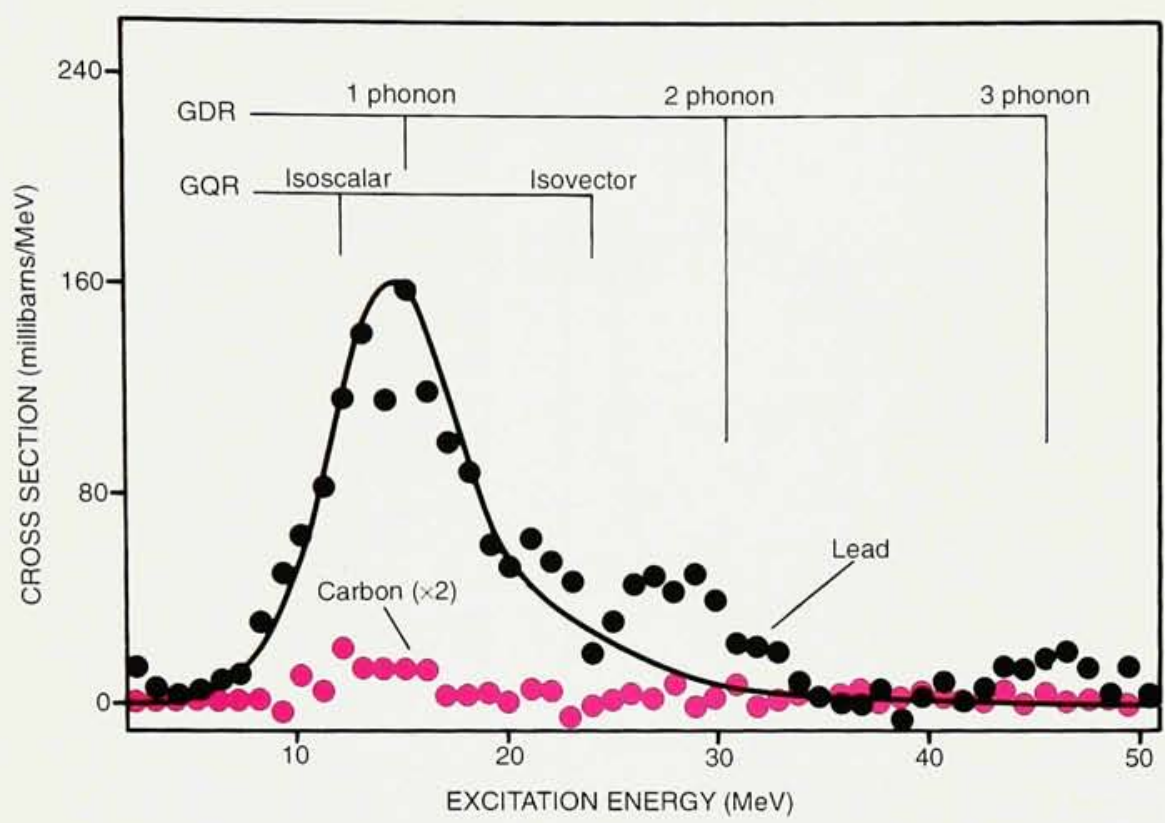

Giant resonance excitation cross sections. Plotted are cross sections for the electromagnetic excitation of the isovector giant dipole resonance (GDR) and of the isoscalar and isovector giant quadrupole resonances $(\mathrm{GQR})$ in $0.7-\mathrm{GeV}{ }^{136} \mathrm{Xe}$ projectiles incident on lead (black points). Also shown are excitation cross sections of two- and three-phonon states. Using carbon targets decreases the cross sections by a factor of 100 (red points). (Adapted from ref. 14.) Figure 5 particle is inversely proportional to the square of the magnetic dipole moment for the transition. Using equation 1 and detailed balance, one can determine the photodecay cross section for the $\Sigma^{0}$ particle. Figure 6 shows the cross sections for the electromagnetic production of $\Sigma^{0}$ on nickel and uranium targets. The theoretical $\mathrm{fit}^{3}$ (solid lines) to these data gives a lifetime of $(7.4 \pm 0.7) \times 10^{-20}$ seconds. This agrees very well with the theoretical predictions and is an important piece of support for the conclusions drawn from the symmetry properties of the SU(3) group.

\section{Production of particles}

As in $\mathrm{e}^{+} \mathrm{e}^{-}$colliders, relativistic heavy ions can be used to investigate photon-photon collisions. (See figure 2c.) In analogy with the one-photon process, described by equation 1 , the photon-photon processes can be related to the real photon processes by ${ }^{18}$

$$
\sigma=\int \frac{\mathrm{d} \omega_{1}}{\omega_{1}} \int \frac{\mathrm{d} \omega_{2}}{\omega_{2}} n_{1}\left(\omega_{1}\right) n_{2}\left(\omega_{2}\right) \sigma_{\gamma \gamma}(\omega)
$$

Here $\omega^{2}=4 \omega_{1} \omega_{2}$. But unlike equation 1, the above formula is only approximate; it is valid for very-highenergy beams and small excitation energy $\hbar \omega$, such

\begin{tabular}{|c|c|c|}
\hline Accelerator & Lorentz factor $\gamma$ & $\begin{array}{c}\text { Cutoff energy } \\
E_{\gamma}^{\max }\end{array}$ \\
\hline GANIL (France) & $1-2$ & $\sim 20 \mathrm{MeV}$ \\
\hline MSU (US) & & \\
\hline RIKEN (Japan) & & \\
\hline GSI (Germany) & $2-3$ & $\sim 50 \mathrm{MeV}$ \\
\hline $\begin{array}{l}\text { AGS at Brookhaven } \\
\text { (US) }\end{array}$ & 15 & $\begin{array}{l}\text { Some } 100 \\
\text { MeV }\end{array}$ \\
\hline $\begin{array}{l}\text { SPS at CERN } \\
\text { (Switzerland) }\end{array}$ & 200 & Some GeV \\
\hline $\begin{array}{l}\text { RHIC (planned at } \\
\text { Brookhaven) }\end{array}$ & $2 \times 10^{4}$ & $\begin{array}{l}\text { Some } 100 \\
\text { GeV }\end{array}$ \\
\hline LHC (planned at CERN) & $3.6 \times 10^{7}$ & $\begin{array}{l}\text { Some } 100 \\
\mathrm{TeV}\end{array}$ \\
\hline
\end{tabular}

The cutoff energies are for heavy-ion collisions. The values of $\gamma$ are given in the reference frame of one of the ions. that the virtual photons are mostly transverse. The interpretation of this formula is that each of the two relativistic ions generates a pulse of photons, $n_{1}$ and $n_{2}$, respectively. The cross section for the photon-photon collisions is given by $\sigma_{\gamma \gamma}$. Integrating over the photon spectrum of each ion yields the cross section for $\gamma \gamma$ collisions in heavy-ion colliders.

An important two-photon process in relativistic heavy-ion colliders is the production of $\mathrm{e}^{+} \mathrm{e}^{-}$pairs. Because the required energy for the process is rather small (about $1 \mathrm{MeV}$ ), the cross sections are huge, on the order of tens of kilobarns for RHIC or hundreds of kilobarns for the LHC. Multiple production of electron pairs would also be possible. The production of $\mathrm{e}^{+} \mathrm{e}^{-}$ pairs could be an important tool for monitoring the beam in the colliders.

Keeping the beam running for a long time could also be a problem. The probability that an electron is created in a bound orbit in one of the ions is not negligible. ${ }^{3}$ This process leads to ion losses from the beam. (The production of free pairs has practically no effect on the beam lifetime.) Estimates of this process indicate that for the high beam luminosities planned for RHIC or the LHC the beam lifetime could be limited

\section{Table 2. Cross sections for electromagnetic} processes

Free $\mathrm{e}^{+} \mathrm{e}^{-}$pairs

Excitation of giant resonances

Bound $\mathrm{e}^{+} \mathrm{e}^{-}$pairs

Excitation of nucleon

resonances

Excitation of multiphonon states

$\pi^{0}$ (two photon)

RHIC
$80 \mathrm{~kb}$
$100 \mathrm{~b}$
$80 \mathrm{~b}$
$20 \mathrm{~b}$
$2 \mathrm{~b}$
$12 \mathrm{mb}$
$5 \mathrm{mb}$
$2 \mathrm{mb}$
$5 \mu \mathrm{b}$
$50 \mathrm{nb}$

LHC

$\eta$

$\eta^{\prime}$

$\eta_{\mathrm{c}}$

$\eta_{\mathrm{b}}$

Higgs boson

$50 \mathrm{nb}$

A uranium-uranium collision is assumed. 


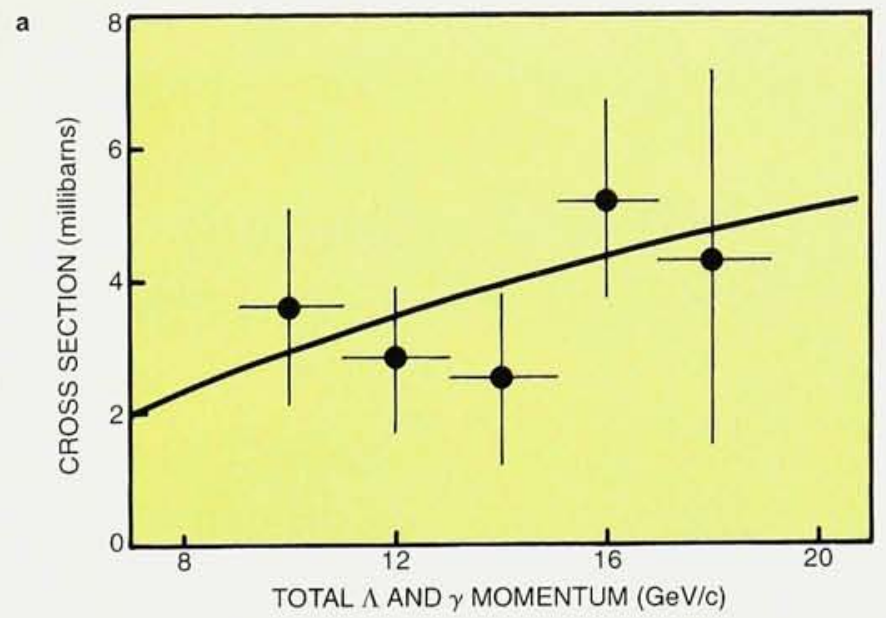

to about 10 hours. ${ }^{19}$

As with the $\mathrm{e}^{+} \mathrm{e}^{-}$colliders, one can study particle production through the two-photon mechanism in heavy-ion colliders. For example, the number of $\pi^{0}$ particles that have been produced up to now at the Hadron-Electron Ring Accelerator at DESY could be created in RHIC in three minutes by the two-photon mechanism. Heavy particles ( $\eta, \eta^{\prime}$, q $\bar{q}$ pairs and so on) could also be produced in this way. ${ }^{18}$ For example, the production of the standard Higgs boson in relativistic colliders by means of the two-photon process would be of the same order as that induced by gluon-gluon interactions. However, the main technique for detecting the Higgs is through its $b \bar{b}$ decay. Because the direct electromagnetic production of $\mathrm{b} \bar{b}$ pairs is larger than that of Higgs bosons, the two-photon mechanism might not be useful in this case. ${ }^{20}$

Table 2 shows the cross sections for several electromagnetic processes that could be studied at RHIC or the LHC. We used $\gamma_{\text {lab }}=100$ at RHIC and $\gamma_{\text {lab }}=4000$ at the LHC. These numbers would be the maximum values for the cross sections, because we assumed that the colliders are filled with uranium beams. If other nuclei are used, one can extract the respective cross sections from these numbers, remembering that one-photon processes scale with $Z^{2}$, while two-photon processes scale with $Z^{4}$. Also, the possibility of measuring these processes depends on the luminosity of the beam as well as on the detection technique used to disentangle the electromagnetic contribution from that originating in hadronic processes.

Not shown in table 2 are many other cases-for example, the cross sections for atomic ionization, which are huge (on the order of kilobarns) and can be very useful for atomic physics studies, or the cross sections for low-energy-photon production, which are also very large and can be useful for beam monitoring. ${ }^{3}$

\section{References}

1. H. R. Schmidt, J. Schukraft, J. Phys. G. 19, 1705 (1993).

2. T. D. Lee, Nucl. Phys. A 538, 3c (1992).

3. C. A. Bertulani, G. Baur, Phys. Rep. 163, 299 (1988).

4. E. Fermi, Z. Phys. 29, 315 (1924). C. F. von Weizsäcker, Z. Phys. 88, 612 (1934). E. J. Williams, Phys. Rev. 45, 729 (1934).

5. J. D. Jackson, Classical Electrodynamics, Wiley, New York (1975).

6. A. Winther, K. Alder, Nucl. Phys. A 319, 518 (1979).

7. W. A. Fowler, Rev. Mod. Phys. 56, 149 (1984).

8. G. Baur, C. A. Bertulani, H. Rebel, Nucl. Phys. A 458, 188 (1986).

9. T. Motobayashi, T. Takei, S. Kox, C. Perrin, F. Merchez, D. Rebreyend, K. Ieki, H. Murakami, Y. Ando, N. M. Iwasa, M. Kurokawa, S. Shirato, J. Ruan (Gen), T. Ichihara, T. Kubo, N. Inabe, A. Goto, S. Kubono, S. Shimoura, M. Ishihara, Phys. Lett. B 264, 259 (1991).

10. J. Kiener, H. J. Gils, H. Rebel, S. Zagromski, G. Gsottschneider, N. Heide, H. Jelitto, J. Wentz, G. Baur, Phys. Rev. C 44, 2195 (1991).

11. K. Ieki, D. Sackett, A. Galonsky, C. A. Bertulani, J. J. Kruse, W. G. Lynch, D. J. Morrisey, N. A. Orr, H. Schulz, B. M. Sherrill, A. Sustich, J. A. Winger, F. Deak, A. Horvath, A. Kiss, Z. Seres, J. J. Kolata, R. E. Warner, D. L. Humphrey, Phys. Rev. Lett. 70, 730 (1993).

12. D. Lissauer et al., proposal for BNL-AGS experiment 814 (accepted October 1985), available at Brookhaven Nat. Lab. library.

13. G. Baur, C. A. Bertulani, Phys. Lett. B 174, 23 (1986).

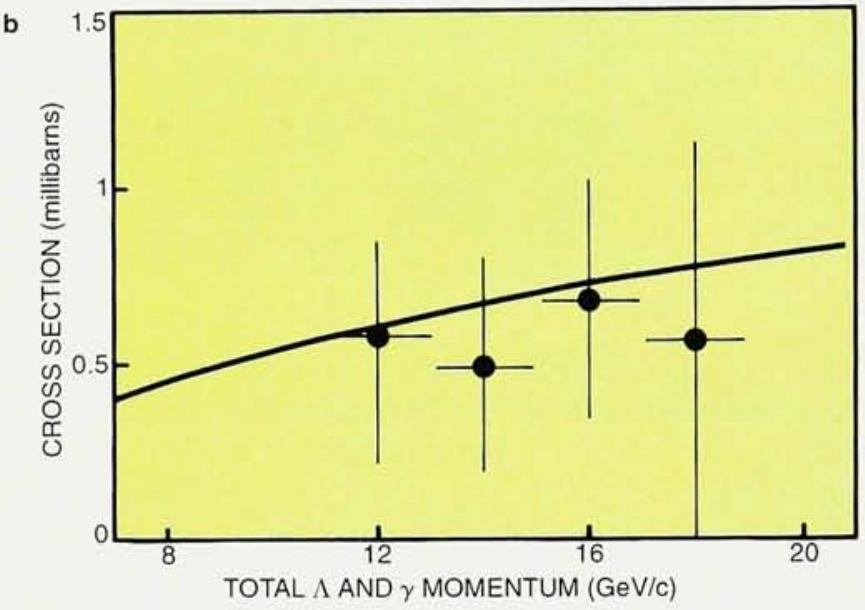

Coulomb production of $\Sigma^{0}$ particles in the field of uranium (a) and nickel (b). ${ }^{16,17}$ The curves are theoretical predictions using the value of $7.4 \times 10^{-20}$ seconds for the lifetime of the $\Sigma^{0}$ particle. (Adapted from ref. 16.)

Figure 6

14. R. Schmidt, Th. Blaich, Th. W. Elze, H. Emling, H. Freiesleben, K. Grimm, W. Henning, R. Holzmann, J. G. Keller, H. Klinger, R. Kulessa, J. V. Kratz, D. Lambrecht, J. S. Lange, Y. Leifels, E. Lubkiewicz, E. F. Moore, E. Wajda, W. Prokopowicz, Ch. Schutter, H. Spies, K. Stelzer, J. Stroth, W. Walus, H. J. Wollersheim, M. Zinser, E. Zude, Phys. Rev. Lett. 70, 1767 (1993)

15. J. Ritman, F.-D. Berg, W. Kuhn, V. Metag, R. Novotny, N. Notheisen, P. Paul, M. Pfeiffer, O. Schwalb, H. Lohner, L. Venema, A. Gobbi, N. Herrmann, K. D. Hildenbrand, J. Mosner, R. S. Simon, K. Teh, J. P. Wessels, T. Wienold, Phys. Rev. Lett. 70, 533 (1993).

16. F. Dydak, F. L. Navarria, O. E. Overseth, P. Steffen, J. Steinberger, H. Wahl, E. G. Williams, F. Eisele, C. Geweniger, K. Kleinknecht, H. Taureg, G. Zech, Nucl. Phys. B 118, 1 (1977).

17. P. C. Petersen, A. Beretvas, T. Devlin, K. B. Luk, G. B. Thomson, R. Whitman, R. Handler, B. Lundberg, L. Pondrom, M. Sheaff, C. Wilkinson, P. Border, J. Dworkin, O. E. Overseth, R. Rameika, G. Valenti, K. Heller, C. James, Phys. Rev. Lett. 57, 949 (1986).

18. G. Baur, C. A. Bertulani, Z. Phys. A 330, 77 (1988)

19. P. Giubelino, in Proc. Conf. Particle Production in Highly Excited Matter, H. H. Gutbrod, J. Rafelski, eds., Plenum, New York (1993), p. 117.

20. K. J. Abraham, M. Drees, R. Laterveer, E. Papageorgiu, A. Schäfer, G. Soff, J. Vermaseren, D. Zeppenfeld, in Proc. ECFA LHC Workshop, vol. II, G. Jarlskog, D. Rein, eds., publ. RD/806-2500, CERN Service d'Information Scientifique, Geneva (February 1991), p. 1224. 